\title{
Importância clínica e laboratorial do volume plaquetário médio
}

\author{
The clinical and laboratory importance of mean platelet volume
}

Mariela Granero Farias'; Suzane Dal Bó

\begin{abstract}
unitermos
Plaquetas

Volume plaquetário médio

Impedância

Densidade ótica

\section{resumo}

Os índices plaquetários fornecidos pelos analisadores hematológicos são provavelmente os parâmetros mais ignorados pela maioria dos laboratórios clínicos, em virtude da dificuldade de sua padronização. Desses índices, o volume plaquetário médio (VPM) vem merecendo destaque por sua grande utilidade, não só em casos de trombose e hemostasia, mas também em uma série de patologias, como diabetes, doenças da tireoide, doenças vasculares, entre outras. O VPM é um parâmetro plaquetário fornecido no hemograma que não gera custos adicionais para o laboratório. Junto com a contagem de plaquetas, ele é um sensível indicador de desordens plaquetárias in vivo, mas pode ser tecnicamente difícil de analisá-lo in vitro por causa dos interferentes pré-analíticos, como tempo de armazenamento da amostra e artefatos gerados pelos anticoagulantes. Neste artigo descrevemos as principais metodologias e seus interferentes na determinação da contagem plaquetária e do VPM, destacando a importância do laboratório de análises clínicas em validar esse parâmetro, proporcionando sua utilização no diagnóstico de desordens hematológicas e de outras patologias.
\end{abstract}

abstract

The platelet indices provided by the hematological analyzers are probably the parameters mostly ignored by most clinical laboratories due to standardization difficulties. Among those indices, the mean platelet volume (MPV) has stood out due to its usefulness not only in cases of thrombosis and hemostasy, but also in a series of pathologies, such as diabetes, thyroid and vascular diseases, among others. MPV is a platelet parameter provided by the hemogram, which does not represent additional costs to the laboratory. Along with platelet count, it is a sensitive indicator of platelet disorders in vivo. However, it may be technically difficult to analyze it in vitro owing to preanalytical interferences, such as sample storage time and anticoagulant artifacts. In this article we described the main methodologies and the interfering factors in determining platelet count and MPV, highlighting the importance of the clinical laboratory analyses to validate this parameter, allowing its use in the diagnosis of hematological disorders and other pathologies. 


\section{Introdução}

As plaquetas foram primeiramente descritas como corpúsculos distintos, e seu papel na coagulação e na trombose foi reconhecido pelo patologista italiano Giulio Bizzozzero, em 1882 ${ }^{(45)}$. Atualmente está bem estabelecida sua definição como fragmentos citoplasmáticos derivados dos megacariócitos da medula óssea ${ }^{(5)}$.

Como as plaquetas não apresentam núcleo, muitas de suas características morfológicas e biológicas são determinadas pela mensagem genética de sua célula precursora, o megacariócito da medula óssea. A trombopoetina (TPO) mostrou-se o maior agente de controle na regulação da produção plaquetária ${ }^{(16,22,32)}$. Esta ação é provavelmente modulada tanto por uma série de citocinas e fatores humorais estimuladores, como as interleucinas (IL) 3, 6 e $11^{(28)}$, quanto por fatores estimulantes de colônia de granulócitos e macrófagos (GM-CSF) e, ainda, pela eritropoetina (EPO). Por outro lado, há os fatores humorais inibidores, como a $\beta$-trombomodulina e o fator plaquetário 4 (PF4), que são proteínas específicas, liberadas dos $\alpha$-grânulos, marcadores da ativação plaquetária, e podem influenciar sua produção ${ }^{(32)}$. Em situações que aceleram a hematopoese, a maturação citoplasmática megacariocítica é mais rápida que a nuclear, e as plaquetas formadas apresentam volume maior e são metabolicamente mais $\operatorname{ativas}^{(60)}$.

Algum tempo atrás, os estudos plaquetários limitavamse, principalmente, à contagem de plaquetas e às referências morfológicas sobre macroplaquetas, satelitismo e agregação plaquetária. Com o surgimento dos analisadores hematológicos de segunda geração, na década de 1980, tornou-se possível a medição automática de vários parâmetros ${ }^{(31)}$. Entre os novos índices, vem merecendo destaque o volume plaquetário médio (VPM), por se tratar de uma variável biológica que determina a função e a atividade plaquetária ${ }^{(46)}$.

São denominadas macroplaquetas aquelas que apresentam um tamanho de 4 a $7 \mu \mathrm{m}$ de diâmetro e plaquetas gigantes as maiores que $7 \mu \mathrm{m}$, geralmente 10 a $20 \mu \mathrm{m}^{(11)}$. Embora a avaliação cuidadosa do tamanho, em uma distensão sanguínea, seja rápida e acurada, ela é subjetiva, uma vez que a morfologia é enganosa para a estimativa do tamanho plaquetário. Plaquetas grandes são mais atrativas que as pequenas; então, frequentemente, quando uma distensão é lida, mostra inadequadamente "plaquetas gigantes"(3). O uso do VPM, determinado pelos analisadores hematológicos, melhorará a descrição de várias desordens plaquetárias (Figura 1).

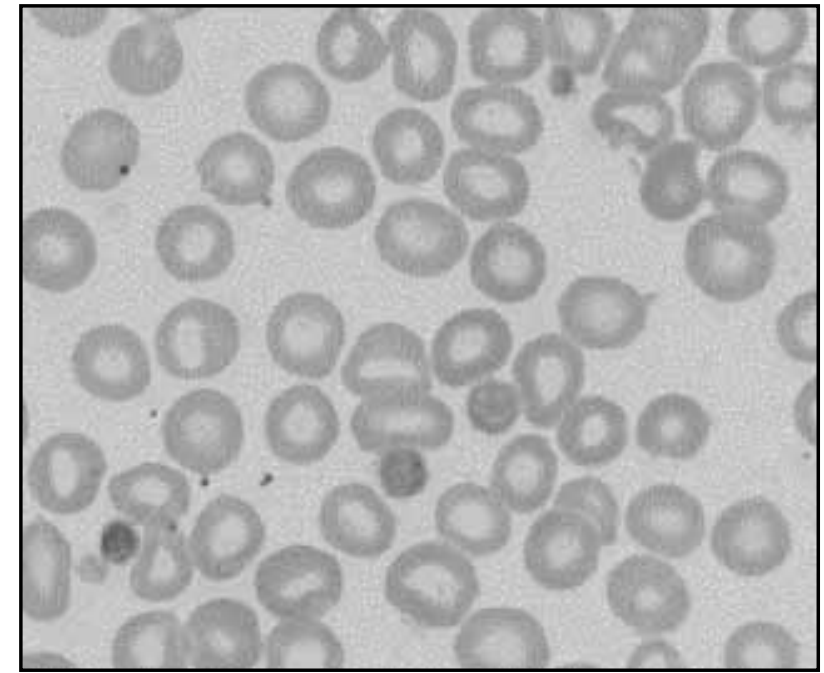

Figura 1 - Distensão sanguínea corada com May-Grünwald-Giemsa (1.000x) mostrando macroplaquetas em um paciente com VPM $12 \mathrm{fL}$

O VPM apresenta relação inversamente proporcional à ploidia do megacariócito. Em face disso, o volume plaquetário não pode ser avaliado separadamente da contagem de plaquetas e uma correlação inversa entre esses parâmetros pode ser encontrada ${ }^{(7,59)}$.

Muitos laboratórios ignoram a informação do VPM, gerada em analisadores hematológicos, pois o método é de difícil padronização e, quando determinado em amostras de rotina, é afetado por numerosas variáveis, como tipo de tecnologia, populações de pacientes e as pertencentes à própria coleta (complexidade dos efeitos dos anticoagulantes e variáveis pré-analíticas, como temperatura e tempo de estocagem do material) ${ }^{(21,53)}$. Em virtude desses problemas e da dificuldade de interpretação da heterogeneidade do tamanho plaquetário sob condições normais e anormais, essas medidas são interpretadas com extrema cautela ${ }^{(35)}$, sendo aconselhável que cada laboratório estabeleça seu próprio valor de referência ${ }^{(15)}$.

A avaliação do tamanho e da morfologia das plaquetas torna-se útil no diagnóstico de pacientes com várias desordens plaquetárias, por isso o VPM é de grande importância, particularmente nas trombocitopenias e trombocitoses. Anormalidades clínicas significativas também podem ser detectadas, mesmo se a contagem de plaquetas estiver nos limites normais ${ }^{(15)}$.

O objetivo deste estudo foi fazer uma revisão bibliográfica do VPM para enfatizar não só a importância deste índice, mas também sua aplicação clínica e laboratorial.

\section{Metodologias}

O primeiro método de referência para a determinação de plaquetas foi a contagem manual por microscopia de 
contraste de fase $\mathrm{e}^{(18,48)}$. Em 2001, o International Council for Standardization in Haematology (ICSH) propôs um novo método, baseado na detecção imunológica por citometria de fluxo, que usa o isotiocianato de fluoresceína (FITC) conjugado com o anticorpo monoclonal $\operatorname{CD} 61^{(8,18,23,34,40)}$. No entanto, os princípios utilizados pela maioria dos analisadores hematológicos continuam sendo a impedância eletrônica e a análise ótica ou a associação das duas metodologias $(4,18,48)$.

A contagem por impedância é baseada na análise dimensional, na qual as plaquetas são identificadas por seu tamanho. As células, incluindo as plaquetas, sequencialmente passam uma após outra através de um orifício aberto; porém, ao passar pelo orifício, alteram uma corrente elétrica que resulta em um sinal. O número de pulsos é proporcional ao de células contadas. O tamanho do pulso elétrico é diretamente proporcional ao tamanho (volume) da célula, o que permite a discriminação específica do tipo de célula com o uso de circuitos threshold ${ }^{(18)}$. O histograma plaquetário é uma curva em que cada plaqueta é classificada e distribuída de acordo com seu tamanho (Figura 2). O VPM é diretamente derivado da análise desta curva, sendo obtido pela divisão do plaquetócrito (PCT) pelo número de plaquetas ${ }^{(5)}$.

No método ótico, o citograma das plaquetas scatter é formado pela medida simultânea de dois diferentes ângulos de dispersão de luz. A dispersão de luz de baixos ângulos ( $2^{\circ}$ a $3^{\circ}$ ) sinalizada é amplificada 30 vezes, e a de altos ângulos $\left(5^{\circ}\right.$ a $\left.15^{\circ}\right), 12$ vezes. $O$ ângulo baixo é convertido em volume celular (tamanho) e o ângulo alto, utilizado para medição do índice de refração (densidade). O citograma mostra plaquetas com volumes de 0 a $30 \mathrm{fL}$. Aquelas que apresentam volume maior que $30 \mathrm{fL}$ são contadas na área dos eritrócitos. A análise integrada é utilizada para distinguir plaquetas, macroplaquetas, eritrócitos, células fragmentadas e debris que tenham diferentes índices de refração $0^{(5,19,50)}$.

A contagem de plaquetas por fluorescência ótica está sendo introduzida com os novos analisadores hematológicos, adicionada à contagem tradicional por impedância. A contagem é realizada no canal de reticuló-

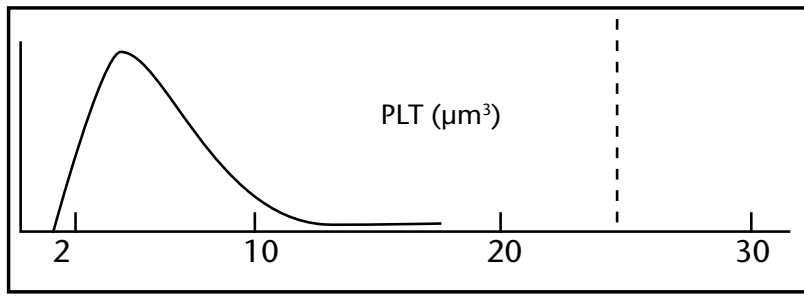

Figura 2 - Histograma de distribuição plaquetária log-normal (Pentra DX 120 ABX) citos. O fuorocromo polimetina marca o conteúdo de RNA/ DNA das células reticuladas e os grânulos e a membrana das plaquetas e produz fluorescência quando excitado com o laser. Essa tecnologia realiza a contagem simultânea de eritrócitos, reticulócitos e plaquetas fluorescentes. A intensidade de fluorescência de cada célula é analisada, o que proporciona a separação das plaquetas dos eritrócitos e reticulócitos. A fluorescência das plaquetas permite não somente a exclusão da contagem de partículas não plaquetárias, mas também a inclusão de macroplaquetas e plaquetas gigantes ${ }^{(5)}$.

Atualmente já existem analisadores hematológicos que utilizam os três princípios, realizando três contagens na mesma diluição: ótica, impedância e imunológica ${ }^{(61)}$.

\section{Problemas tecnológicos na determinação do VPM}

Embora o ácido etilenodiaminotetracético (EDTA) seja o anticoagulante de rotina, mais utilizado para contagem de células sanguíneas, ele pode induzir alterações na forma e na ultraestrutura das plaquetas. Plaquetas coletadas com esse anticoagulante alteram-se com o tempo e a temperatura ${ }^{(6,39)}$, tornando-se esféricas. Entretanto, quando coletadas com citrato de sódio, tornam-se discoides $^{(6,26,38)}$. Essa alteração na forma causa aumento aparente de aproximadamente $20 \%$ no VPM nas duas primeiras horas em exposição ao EDTA, em relação às amostras coletadas com citrato( $^{(38)}$.

Estudos anteriores relatam que o valor do VPM aumenta em decorrência do inchaço plaquetário. Entretanto, um estudo de Endler et al. mostra que tal aumento de tamanho é, aproximadamente, inferior a $0,5 \mathrm{fL}$, quando a análise é realizada em até duas horas após a venipuntura ${ }^{(17)}$.

A pseudossíndrome de plaquetas cinzentas é um raro fenômeno dependente do EDTA que causa a degranulação das plaquetas in vitro, gerando uma aparência cinza no sangue periférico. As plaquetas "fantasmas" resultam na diminuição da contagem plaquetária e na elevação do VPM. Valores diminuídos de VPM podem ser obtidos pelo estouro das plaquetas, também causado pelo EDTA ${ }^{(36)}$.

Na metodologia de impedância, o tamanho é o principal parâmetro para separar plaquetas de eritrócitos ou de outras partículas, como, por exemplo, células vermelhas fragmentadas, micrócitos, fragmento de células leucêmicas de leucemias agudas ou crônicas ${ }^{(8,13,29,30,51)}$, crioglobulinas ${ }^{(13,14,54)}$, ou qualquer outra causa que gere a formação de debris (Figura 3 a-c). Como todas as partículas com 
menos de $20 \mathrm{fL}$ podem ser contadas como plaquetas, a contagem fica espuriamente elevada e o VPM fica alterado, refletindo o tamanho do fragmento ${ }^{(29,30)}$. Eritrócitos infectados por trofozoítos do P. falciparum ${ }^{(13)}$, por fungos ${ }^{(1,29)}$ ou bactérias ${ }^{(1,30)}$ também podem interferir na contagem de plaquetas e na determinação do VPM.

As bactérias podem induzir contagens falsamente elevadas de plaquetas por sua presença in vivo. Embora seja uma situação rara, em pacientes sépticos algumas bactérias podem ser observadas na distensão sanguínea, associadas a culturas positivas. $\mathrm{O}$ histograma do volume plaquetário é anormal e mostra um pico de partículas de pequeno tamanho (2 fL ou menos) quando há bactérias ou aglomerados bacterianos. Fungos também podem apresentar o tamanho das plaquetas e ser observados na distensão sanguínea. Recentemente foi relatada uma contagem elevada de plaquetas em um paciente trombocitopênico infectado por Candida ${ }^{(56)}$.

Plaquetas muito grandes e agregados plaquetários apresentam maiores valores para VPM e contagens diminuídas ${ }^{(1,8,24,44)}$, uma vez que são registrados a partir de $20 \mathrm{fL}$ (Figura 3 d-e).

Em pacientes com hiperlipidemia, em amostras coletadas após refeições ou nutrição parenteral, os lipídios podem formar pequenas gotas in vitro, que alteram a contagem de plaquetas e causam moderado aumento em analisadores que usam o método ótico. Como os lipídeos possuem alto índice refratário, eles podem gerar sinais anormais localizados próximo das plaquetas ou no mesmo local ${ }^{(56)}$.

\section{Aplicação clínica}

O VPM é correlacionado com a função plaquetária e pode ser um índice mais sensivel que o número de plaquetas, como marcador de interesse clínico, em várias desordens.

O volume plaquetário elevado pode ser encontrado em: pré-eclâmpsia ${ }^{(46)}$, diabetes ${ }^{(25,43,59)}$, doenças cardía$\operatorname{cas}^{(20,27,58)}$, hipertireoidismo ${ }^{(15)}$, esplenectomia ${ }^{(3)}$, talassemias $\alpha$ ou $\beta^{(3)}$, vírus da imunodeficiência humana $(\mathrm{HIV})^{(42)}$ e púrpura trombocitopênica idiopática (PTI) ${ }^{(10,12,37)}$. Em desordens mieloproliferativas, como a leucemia mieloide crônica e a mielofibrose, as plaquetas frequentemente, mas não sempre, são grandes e anormalmente heterogêneas. Nessas doenças, realmente há plaquetas "gigantes" no sangue periférico ${ }^{(44)}$. A trombocitemia essencial e a policetemia vera não estão associadas a plaquetas anormais e grandes. Nessas desordens, a medula óssea é hiperproliferativa, mas as células resultantes são fenotipicamente normais(3). Plaquetas grandes são vistas também em várias causas de trombocitopenias congênitas, como a síndrome de Bernard-Soulier e a anomalia de May-Hegglin. Nesta também

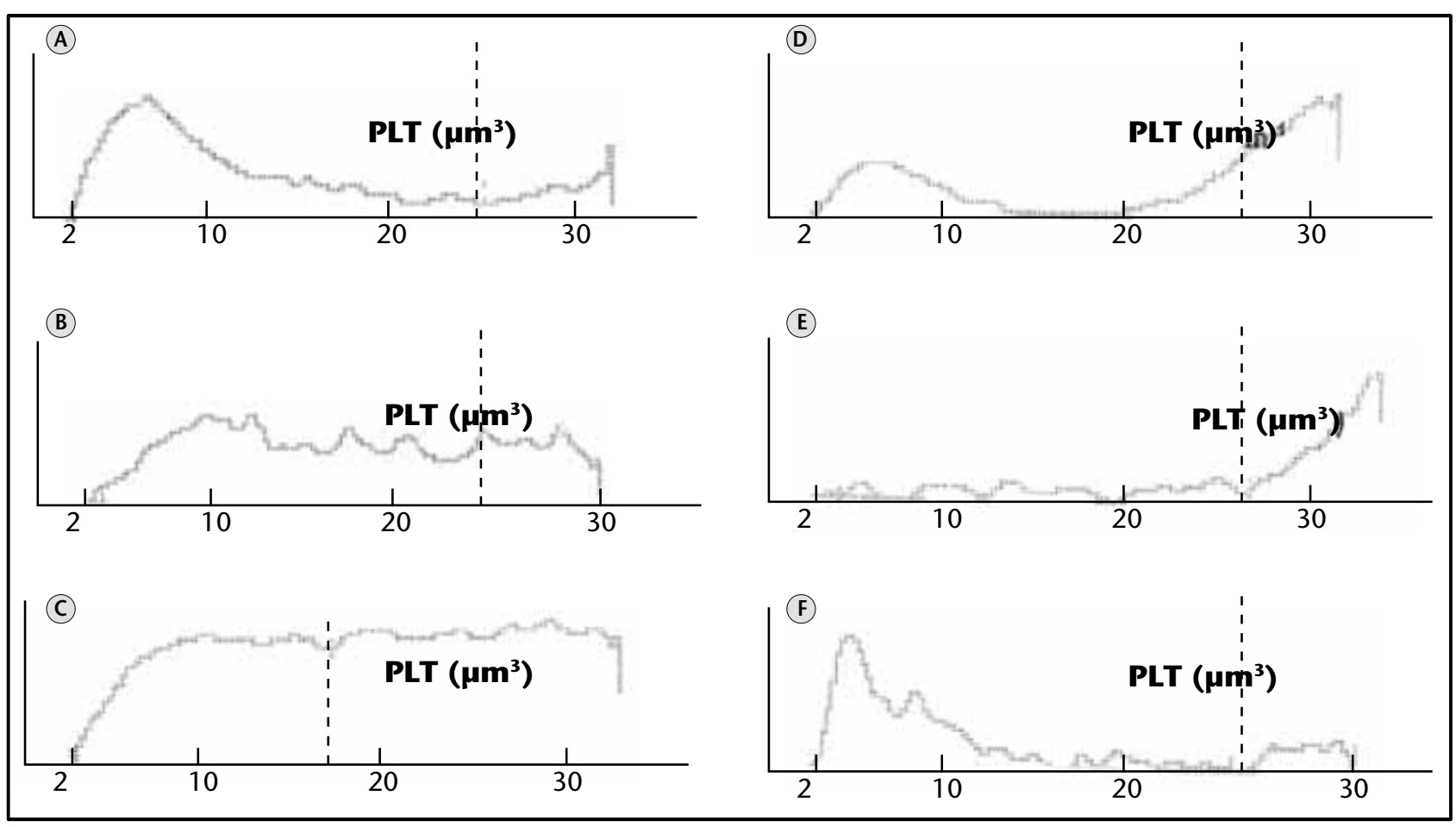

Figura 3 - Histogramas plaquetários mostrando interferentes: eritrócitos fragmentados $(\boldsymbol{A}, \boldsymbol{B})$, micrócitos $(\boldsymbol{C})$, macroplaquetas $(\boldsymbol{D}, \mathbf{E})$, fragmentos de células leucêmicas (F) (Pentra DX 120 ABX) 
podem ser vistas plaquetas gigantes no sangue periférico. Na rara síndrome de plaquetas cinzentas, elas são grandes e agranuladas ou hipogranuladas, em virtude da liberação de

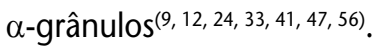

O volume plaquetário diminuído pode ser encontrado em: sepse ${ }^{(55)}$, quimioterapia ${ }^{(3)}$, doenças inflamatórias do intestino, como colite ulcerativa e doença de $\mathrm{Crohn}^{(32)}$, hipotireoidismo ${ }^{(3)}$, anemia megaloblástica ${ }^{(3)}$ e síndrome de Wiskott-Aldrich ${ }^{(51)}$. Nessa rara síndrome hereditária, a trombocitopenia é acompanhada por plaquetas pequenas e hipofuncionais $(9,12,37,49,51)$.

\section{Discussão}

Por muito tempo, os estudos referentes a plaquetas relacionavam-se somente a trombose e hemostasia. Atualmente está claro que as plaquetas exercem importante papel em diversas desordens caracterizadas por doenças vasculares, incluindo doença arterial coronariana (DAC), doenças mieloproliferativas, diabetes, pré-eclâmpsia, doença intestinal inflamatória, entre outras ${ }^{(20)}$.

Em pacientes com diabetes têm sido relatadas alterações na morfologia e na função plaquetária. A hiperatividade plaquetária é acompanhada por aumento na síntese de tromboxano e/ou por diminuição na produção de prostaciclina ${ }^{(25)}$. Plaquetas grandes são mais reativas e agregáveis, porque contêm grânulos mais densos que secretam mais serotonina, $\beta$-trombomodulina e produzem mais tromboxano A2. Isso sugere uma relação entre a função plaquetária e as complicações diabéticas. Alguns estudos têm mostrado uma vida curta e rápida de plaquetas em diabéticos ${ }^{(25)}$.

Plaquetas grandes, na circulação, refletidas pela elevação do VPM, levam à maior agregação e facilitam a formação do trombo ${ }^{(20,57,58,62)}$, o que mostra ser um fator de risco independente para o infarto do miocárdio $(\mathrm{IM})^{(2)}$ e para o aparecimento da obstrução arterial coronariana na angina instável(20, 27, 32, 56). Um aumento do VPM seis meses após o IM é associado a maior risco de futuro infarto ${ }^{(27,56)}$. Pacientes que tiveram IM, ou em risco, necessitam de VPMs seriados, bem como de enzimas e outros marcadores ${ }^{(15)}$.

O VPM apresenta relação inversamente proporcional à ploidia do megacariócito; assim, o volume plaquetário não pode ser avaliado separadamente da contagem de plaquetas e uma correlação inversa entre eles pode ser encontrada ${ }^{(7,62)}$. Entre as citocinas mais relevantes, a IL-3, a TPO e a IL-6 têm maior influência na ploidia do megacariócito, o que leva à produção de plaquetas grandes e mais reativas ${ }^{(17)}$.

Uma das razões mais importantes para as contagens automatizadas é o baixo erro estatístico devido ao grande número de células examinadas. Entretanto, essas tecnologias apresentam limitações que podem afetar uma contagem verdadeira de plaquetas. Tais limitações são causadas pela inabilidade em diferenciá-las de partículas interferentes ${ }^{(18)}$. A interferência do anticoagulante deve ser controlada pelo uso de um anticoagulante alternativo ou pela padronização entre o tempo da coleta e a análise da amostra ${ }^{(5)}$.

Aumento espúrio da contagem de plaquetas também é relatado em várias situações, incluindo células vermelhas fragmentadas, crioglobulinas, bactérias ou fungos e lipídios. Alarmes (flags) são gerados e alertam o operador quanto a possíveis achados anormais, podendo-se, assim, identificar o problema. O operador deve estar ciente das características do seu analisador hematológico e apto a reconhecer e corrigir resultados anômalos.

O histograma do volume de distribuição plaquetária é de particular importância. Quando ele for de forma log-normal, a contagem de plaquetas e o VPM refletirão valores reais. Em contraste, quando a distribuição for log-anormal, tanto a contagem quanto o VPM estarão representando interferentes. A avaliação microscópica da lâmina, embora menos precisa, também é de grande utilidade para a identificação de alterações ${ }^{(1,13,29,30)}$.

A confirmação, usando outro método de contagem, como o de referência - a citometria de fluxo -, deve ser realizada ao menos nas seguintes circunstâncias: falha na curva de plaquetas, na base de $20 \mathrm{fL}$, população de micrócitos presentes no histograma e VPM exageradamente alto(56).

O VPM é gerado a partir de analisadores hematológicos, como parte integrante do hemograma, por isso não implica custos adicionais para o laboratório. Tal índice, quando associado à contagem de plaquetas, permite duas vezes mais a detecção de anormalidades plaquetárias, sendo particularmente importante no diagnóstico e no controle de desordens hematológicas e em uma série de outras patologias. 


\section{Referências}

1. BAIN, B. J.; PATH, F. R. C. Diagnosis from the blood smear. N Engl J Med, v. 353, n. 5, p. 498-507, 2005.

2. BATH, P. et al. Association of mean platelet volume with risk of stroke among 3134 individuals with history of cerebrovascular disease. Stroke, v. 35, p. 622-6, 2004.

3. BESSMAN, J. D. Automated blood counts and differentials: a pratical guide. Johns Hopkins University Press, 1986. p. 57-83.

4. BRIGGS, C. et al. Continuing developments with the automated platelet count. Int Jnl Lab Hem, v. 29, p. 77-91, 2007.

5. BRIGGS, C. et al. New quantitative parameters on a recently introduced automated blood cell counter: the XE 200TM. Clin Lab Med, v. 22, p. 345-50, 2000.

6. BRUMMITT, D. R.; BARKER, H. F. The determination of a reference range for new platelet parameters produced by the Bayer ADVIATM 120 full blood count analyser. Clin Lab Haem, v. 22, p 103-7, 2000.

7. BUTKIEWICZ, A. M. et al. Platelet count, mean platelet volume and thrombocytopoietic indices in healthy women and men. Thromb Res, v. 118, n. 2, p. 199-204, 2006.

8. CHARIE, L. A. et al. Accuracy in the low platelet count range: a comparison of automated platelet count on beckman coulter high-volume hematology analyzers with the ISLH/ICSH platelet reference method. Lab Haem, v. 7, p. 236-44, 2001.

9. CINES, D. B. etal. Congenital and acquired thrombocytopenia. Hematology, v. 2004, p. 390-406, 2004.

10. CINES, D. B.; BLANCHETTE, V. Immune thrombocytopenic purpura. N Engl J Med, v. 346, n. 13, p. 995-1008, 2002.

11. COLLEGE OF AMERICAN PATHOLOGISTS. SURVEYS \& ANATOMIC PATHOLOGY EDUCATION PROGRAMS. Hematology, clinical microscopy and body fluids glossary. 2005. p. 11.

12. CORCORAN, G.; KOTTKE-MARCHANT, K. Chasing after the causes of platelet disorders. Arch Pathol Lab Med, v. 126, p. 133-46, 2002.

13. CRABBE, G. et al. Artefactually-normal automated platelet count due malaria-infected RBC. Clin Lab Haem, v. 24, p. 179-82, 2002.

14. DELGADO, J. et al. Cryoglobulinemia detected as a PIC/ POC discrepancy of the automated complete blood count. Eur J Haematol, v. 69, p. 65-6, 2002.

15. DOW, R. B. The clinical and laboratory utility of platelet volume parameters. Aus J Med Sci, v. 15, p. 1-18, 1994.

16. DRACHMAN, J. G. Inherited thrombocytopenia: when a low platelet count does not mean ITP. Blood, v. 103, n. 2, p. 390-8, 2004.

17. ENDLER, G. et al. Mean platelet volume is an independent risk factor for myocardial infarction but not for coronary artery disease. Br J Haematol, v. 117, p. 399-404, 2002.

18. FELLE, P. et al. Platelets in the paediatric population: the influence of age and the limitations of automation. Clin Lab Haem, v. 27, p. 250-7, 2005.
19. GIACOMINI, A. et al. Platelet count and parameters determined by te Bayer ADVIATM 120 in reference subjects and patients. Clin Lab Haem, v. 23, p. 181-6, 2001.

20. GREISENEGGER, S. et al. Is elevated mean platelet volume associated with a worse outcome in patients with acute ischemic cerebrovascular events? Stroke, v. 35, p. 1688-91, 2004.

21. GULATI, G. L. et al. Changes in automated complete blood cell count and differential leukocyte count results induced by storage of blood at room temperature. Arch Pathol Lab Med, v. 126, p. 336-42, 2002.

22. HARKER, L. A. et al. Effects of megakaryocyte growth and development factor on platelet production, platelet life span, and platelet function in healthy human volunteers. Blood, v. 95, p. 2514-22, 2000.

23. HARRISON, P. et al. Immunoplatelet counting: a proposed new reference procedure. Br J Haematol, v. 108, p. 228-35, 2000.

24. HATZIPANTELIS, E. S. et al. Hereditary giant platelet disorder presented as pseudothrombocytopenia. Eur J Haematol, v. 67, p. 330-1, 2001.

25. HEKIMSOY, Z. et al. Mean platelet volume in type 2 diabetic patients. J Diabetes Complications, v. 18, p. 173-6, 2004.

26. HENRY, J. B. Clinical diagnosis and management by laboratory methods. 20. ed. Philadelphia: W.B. Saunders, 2001. p. 448.

27. HUCZEK, Z. et al. Mean platelet volume on admission predicts impaired reperfusion and long-term mortality in acute myocardial infarction treated with primary percutaneos coronary intervention. JACC, v. 46, n. 6 , p. 284-90, 2005.

28. ITALIANO JR, J. E.; SHIVDASANI, R. A. Megakaryocytes and beyond: the birth of platelets. J Tromb Haemost, v. 1, p. 1174-82, 2003.

29. KAITO, K. et al. Platelet size deviation width, platelet large cell ratio, and mean platelet volume have sufficient sensivity and especificity in the diagnosis of immune trombocytopenia. Br J Haematol, v. 128, p. 698-702, 2005.

30. KAKKAR, N. Spurius rise in the automated platelet count because of bacteria. J Clin Pathol, v. 57, p. 1096-7, 2004.

31. KAKKAR, N.; GARG, G. Cytoplasmatic fragments of leukaemic cells masquerading as platelets in an automated haematology analyser. J Clin Pathol, v. 58, p. 224, 2005.

32. KAPSORITAKIS, A. N. et al. Mean platelet volume: a useful marker of inflammatory bowel disease activity. Am J Gastroenterol, v. 96, p. 776-81, 2001.

33. KUNISHIMA, S.; SAITO, H. Congenital macrothrombocytopenias. Blood Rev, v. 20, n. 2, p. 111-21, 2006.

34. KUNZ, D. et al. Automated CD61 immunoplatelet analysis of thrombocytopenic samples. Br J Haematol, v. 112, p. 584-92, 2001. 
35. LEE, G. R. et al. Wintrobe hematologia clínica. 1. ed. São Paulo: Manole, 1998.

36. LESESVE, J. F. et al. Pseudo-storage pool disease due to platelet degranulation in EDTA-collected peripheral blood: a rare artifact. Clin Lab Haem, v. 27, p. 336-42, 2005.

37. LEUNG, A. K. C.; CHAN, K. W. Evaluating the child with purpura. Am Fam Physician, v. 64, p. 419-28, 2001.

38. MACEY, M. et al. Evaluation of the anticoagulants EDTA and citrate, theophylline, adenosine and dipyridamole (CTAD) for assessing platelet activation on the Advia 120 hematology system. Clin Chem, v. 48, n. 6, p. 891-9, 2002.

39. NISHIOKA, T. et al. Flow cytometric analysis of platelet activation under calcium ion-celating conditions. Clin Lab Haem, v. 24, p. 115-9, 2002.

40. NORRIS, S. et al. Immunoplatelet counting: potential for reducing the use of platelet transfusions through more accurate platelet counting. Br J Haematol, v. 131, p. 605-13, 2003.

41. PÉREZ-PUJOL, S. etal. Glycoproteins expression on platelet membrane in inherited macrothrombocytopenias. Thromb Res, v. 112, p. 233-7, 2003.

42. PUYO, C. A. Thrombocytopenia. Int Anesthesiol Clin, v. 39, n. 1, p. 17-34, 2001.

43. QUINTANA, X. G. et al. Las plaquetas en la diabetes mellitus. Rev Cubana Hematol Inmunol Hemoter, v. 17, n. 1, p. 19-24, 2001.

44. RACCHI, O.; RAPEZZI, D. Megathrombocytes and spurious thrombocytopenia. Eur J Haematol, v. 66, p. 140-1, 2001.

45. RIBATTI, D.; CRIVELLATO, E. Giulio Bizzozero and the discovery of platelets. Leuk Res, v. 31, p. 1339-41, 2007.

46. SANTOS, E. V.; MEIRELLES FILHO, J. Plaquetograma em gestantes normais e com pré-eclâmpsia. RBGO, v. 26, n. 3, p. 201-6, 2004.

47. SAVolA, A. et al. Autosomal dominant macrothrombocytopenia in Italy is most frequently a type of heterozygous Bernard-Soulier syndrome. Blood, v. 97, n. 5, p. 1330-5, 2001.

48. SEGAL, H. C. et al. Accuracy of platelet counting haematology analysers in severe thrombocytopenia and potential impact on platelet transfusion. Br J Haematol, v. 128, p. 520-5, 2005.

49. SHAPIRO, A. D. Platelet function disorders. Haemophilia, v. 6, n. 1, p. 120-7, 2000.

50. STANWORTH, S. J. et al. Automated counting of platelets on the Bayer ADVIATM 120 analyser. Clin Lab Haem, v. 21, p. 113-7, 1999.

51. THRASHER, A. J.; KINNON, C. The Wiskott-Aldrich syndrome. Clin Exp Immunol, v. 120, p. 2-9, 2000.

52. VAN DER MEER, W. et al. Pseudoplatelets: a retrospective study of their incidence and interference with platelet counting. J Clin Pathol, v. 56, p. 772-4, 2003.

53. VOGELAAR, S. A. et al. Blood stability at room temperature for counting red and white blood cells and platelets. Vascul Pharmacol, v. 39, p. 123-5, 2002.

54. VON AHSEN, N. et al. Cryoglobulins interfere with platelet counts by optical and impedance methods but not with the CD61 immunoplatelet count. Clin Chem, v. 10, p. 1858-60, 2001.

55. YAGUCHI, A. et al. Platelet function in sepsis. J Thromb Haemost, v. 2, p. 2096-102, 2004.

56. YANG, A. et al. Mean platelet volume as marker of restenosis after percutaneos transluminal coronary angioplasty in patients with stable and unstable angina pectoris. Thromb Res, v. 117, p. 371-7, 2006.

57. YILMAZ, M. B. et al. Mean platelet volume and exercise stress test. J Thromb Thrombolysis, p. 115-20, 2004.

58. YILMAZ, M. B. et al. Platelet aggregation in left ventricular thrombus formation after acute anterior myocardial infarction: mean platelet volume. Int J Cardiol, v. 90, p. 123-5, 2003.

59. YNGEN, M. Platelet hyperactivity in diabetes mellitus. Business briefing: European Cardiology, p. 1-6, 2005. Disponível em: <http://www.touchcardiology.com/ platelet-hyperactivity-diabetes-mellitus-a370-1.html>.

60. ZAGO, M. A. et al. Hematologia: fundamentos e prática. 1. ed. São Paulo: Atheneu, 2004. p. 735.

61. ZANDECKI, M. et al. Spurius counts and spurious results on haematology analysers: a review. Part I: platelets. Int Jnl Lab Hem, v. 29, p. 4-20, 2007.

62. ZIMMET, J.; RAVID, K. Polyploidy: occurrence in nature, mechanisms, and significance for the megakaryocyteplatelet system. Exp Hematol, v. 28, p. 3-16, 2000. 